\title{
Archives
}

\section{La Division de la gestion de documents et des archives de l'Université de Montréal et l'Association des archivistes du Québec : des chemins croisés}

\section{CATHERINE DUGAS}

Volume 46, numéro 2, 2017

$50^{\mathrm{e}}$ anniversaire de la DGDA

URI : https://id.erudit.org/iderudit/1040386ar

DOI : https://doi.org/10.7202/1040386ar

Aller au sommaire du numéro

Éditeur(s)

Association des archivistes du Québec (AAQ)

ISSN

0044-9423 (imprimé)

2369-9256 (numérique)

Découvrir la revue

Citer cet article

Dugas, C. (2017). La Division de la gestion de documents et des archives de l'Université de Montréal et l'Association des archivistes du Québec : des chemins croisés. Archives, 46(2), 133-143. https://doi.org/10.7202/1040386ar
Résumé de l'article

Une association telle que l'Association des archivistes du Québec ne peut croître sans l'apport de bénévoles dynamiques et dévoués. À travers le temps, plusieurs de ses acteurs importants ont été des archivistes employés de la Division de la gestion de documents et des archives de l'Université de Montréal. Cette présentation démontrera l'éventail des personnalités qui ont marqué l'histoire et contribué au développement de ces deux institutions. 


\section{$50^{\mathrm{E}}$ ANNIVERSAIRE DE LA DGDA}

\section{La Division de la gestion de documents et des archives de I'Université de Montréal et l'Association des archivistes du Québec: des chemins croisés}

\section{CATHERINE DUGAS}

Représentante de I'Association des archivistes du Québec

Ce texte porte sur les liens entre la Division de la gestion de documents et des archives de I'Université de Montréal (DGDA) et l'Association des archivistes du Québec (AAQ). Jolie coïncidence, la DGDA fête ses 50 ans seulement quelques mois avant le déclenchement des festivités du $50^{\mathrm{e}}$ anniversaire de l'AAQ.

II aurait été possible d'énumérer simplement tous les anciens du service qui se sont impliqués à l'AAQ et dans le milieu archivistique élargi puisque, dès les débuts de l'association, plusieurs des acteurs importants de sa constitution se retrouvent également dans la longue liste des employés de la DGDA. Pour avoir la chance de faire plus qu'un exercice chronologique, nous soulignerons ici certains des bénévoles de la DGDA qui ont marqué I'histoire de l'AAQ et auxquels nous sommes redevables pour avoir amené I'association jusqu'à sa forme actuelle. 


\section{AUX DÉBUTS DE L'AAQ}

II faut se rappeler qu'au début des années 1960, aucune association n'existe spécifiquement pour les archivistes québécois. Ceux-ci se rencontrent, entre autres, lors des activités de la Society of American Archivists. Le congrès de cette association en octobre 1967 est un moment décisif. Sur 288 participants (Society of American Archivists, 2016), quelques archivistes québécois s'y rencontrent et se disent que le moment est venu pour se regrouper localement considérant que des discussions sur le sujet ont lieu depuis 1963. Luc-André Biron, premier directeur de la DGDA (1966-1971), et François Beaudin, second directeur de la DGDA (1972- 1976), font partie de ces visionnaires (Université de Montréal. Division de la gestion de documents et des archives, 2010). Dès leur retour au Québec, les idées bouillonnent et le 9 décembre 1967 a lieu la première rencontre officielle de I'Association des archivistes du Québec (Gagnon-Arguin, 1992, p. 125-126). Un comité provisoire est mis sur pied; François Beaudin y est nommé vice-président et est chargé de présenter un projet de constitution pour la prochaine rencontre. Robert Garon le rapporte dans un texte de 1974 publié dans la revue Archives: «Les archivistes devaient s'unir pour participer, comme tous les autres Québécois, à la renaissance nationale: il importait de fournir un apport à l'émancipation collective, il importait également d'en tirer profit» (p.5).

La première année, 125 membres adhèrent à l'association et, dès le départ, ses membres ont conscience du besoin de s'impliquer dans cette nouvelle entité créée pour soutenir la communauté archivistique (GagnonArguin, 1992, p. 10). Les débuts de l'AAQ sont bien sûr consacrés à la mise en place d'une structure administrative dont Luc-André Biron fait partie en qualité de conseiller au conseil d'administration de 1970 à 1973. François Beaudin y joue également un rôle central en tant que vice-président, président et président sortant de 1968 à 1971 et à nouveau de 1974 à 1976 comme vice-président et président. Signe d'un vrai visionnaire, dans son rapport de président sortant en 1976, François Beaudin critique le manque de membres féminins sur le conseil d'administration et note qu'il serait plus que temps d'avoir une vice-présidente ou présidente (Beaudin, 1976).

Une des priorités lors de la mise en place de cette nouvelle association est d'avoir une force d'action pour assurer une formation archivistique de qualité aux archivistes déjà en place et aux futurs archivistes. Un comité de formation est donc instauré. François Beaudin y est un membre 
important de 1971 à 1974 et y tient la présidence en 1972. Le comité met sur pied des cours d'archivistique au niveau collégial allant même jusqu'à recruter les professeurs, des discutions commencent aussi pour des cours de niveau universitaire (Comité de formation de I'Association des archivistes du Québec, 1971).

Des publications sont également créées pour permettre une meilleure circulation des idées. La revue Archives voit le jour dès 1969. Luc-André Biron y participe en tant que bénévole pour la cueillette et la mise en dactylo des textes, mais il y publie aussi; notamment un texte intitulé Pourquoi je suis archiviste qui reste frappant d'actualité même si publié en 1972. Regrettant le manque de formation en gestion des archivistes, il pointe cette caractéristique comme une nécessité inébranlable du métier même si «l'archiviste apparaît aux yeux de tout le monde, comme une "vieille barbe», personnage plutôt cinglé, miteux, désagréable, poussiéreux, dépassé »(Biron, 1972).

La Chronique suit en 1971 pour alléger la tâche de la revue en se concentrant sur les nouvelles associatives et les nouvelles des membres. Jean-Yves Rousseau, directeur du Service des archives pendant une décennie (1988-1998), est membre du comité de La Chronique dans les années 70 et y brigue la présidence en 1974.

L'association désire offrir un rendez-vous annuel à ses membres. Les congrès commencent donc à se tenir dès 1972 et François Beaudin préside le comité organisateur d'un des premiers congrès en 1974. Le thème de ce congrès Perspectives 1980 cherche à porter un regard vers l'avenir qui attend les archivistes. Les intitulés des ateliers, Généalogistes et archivistes - Un duel ou un duo? ou Le Microfilm - un luxe indispensable?, démontrent les problématiques de l'époque (Association des archivistes du Québec, 1975).

Un moment important de I'histoire de l'AAQ est son incorporation en vertu de la Loi sur les compagnies. François Beaudin est un des signataires des lettres patentes ainsi que le président du Comité des structures de 1974 à 1975, comité qui travaille à réviser les règlements de l'AAQ dans le but de son incorporation. Le comité présente aussi un rapport sur le partage des tâches entre le conseil d'administration et le comité exécutif qui comprend même une section sur les devoirs des membres individuels (Conseil de l'Association des archivistes du Québec, 1974). 
Le milieu des années 70 voit aussi l'arrivée de sections distinctives à I'intérieur de l'association. Les archivistes religieux composent l'effectif de l'association avec une portion grandissante d'archivistes travaillant avec les archives «vivantes»; chacun de ces groupes se sent lésé dans les services offerts. En réponse, les sections Archives historiques et Gestion des documents sont créées en 1974 avec des comités dans différentes régions.

Le conseil d'administration témoigne de l'implication d'autres employés de la DGDA. Mentionné précédemment, Jean-Yves Rousseau est trésorier de l'AAQ (1975-1976), vice-président (1983-1984) et également président du comité de formation au début des années 80 (1982). Claude Minotto, directeur du Service de 1999 à 2009, est secrétaire du conseil à la fin des années 70 (1977-1978). À cette époque, Denise Pélissier, directrice intérim de la DGDA de 1998 à 1999, est secrétaire et membre correspondant (1979-1981) pour la revue Archives. Elle participe également à la Section archives historiques, région Montréal (1976-1977).

\section{L'ADOLESCENCE DE L'AAQ}

Les années 80 voient une continuation des activités de l'AAQ avec un travail en amont dans le cadre du projet de la Loi sur les archives qui sera adoptée en 1983. En 1981, I'AAQ reconnaît la contribution spéciale au fonctionnement de l'association de François Beaudin en lui remettant le prix du membre émérite (AAQ membre émérite, 1981). La recherche de partenariats avec des organismes de différents niveaux, provincial, fédéral et international, se poursuit. Ce désir de collaboration est facilité par le fait que les membres de l'AAQ sont déjà actifs dans d'autres organisations. Par exemple, François Beaudin participe à la Section archives de la Société historique du Canada. Plusieurs membres de la DGDA forment même d'autres groupes. Le Groupe d'archivistes de la région de Montréal (GARM) voit le jour en 1982 pour accentuer la collaboration entre les services d'archives de la région. Le Groupe interdisciplinaire de recherche en archivistique (GIRA) est une initiative de Jacques Ducharme, Jean-Yves Rousseau et Carol Couture en 1987, qui a pour objectif de stimuler la réflexion et d'encadrer la recherche. L'association offre ainsi un lieu de discussion professionnelle qui «traverse les lignes institutionnelles» comme le mentionne Claude Minotto dans le bilan de son mandat de secrétaire (Minotto, 1978). 
Le cadre légal offert par la Loi sur les archives ne rend pas nécessairement la tâche plus facile pour l'AAQ et ses administrateurs. La nécessité de regroupement reste forte pour contrer la concurrence d'autres professions émergentes dans le contexte de l'informatisation des bureaux. La structuration du milieu se continue et les membres de la DGDA via I'AAQ sont encore une fois des acteurs importants, que ce soit dans la mise sur pied du Conseil canadien des archives ou le Bureau canadien des archivistes (Conseil de l'Association des archivistes du Québec, 1974).

De nouveaux membres prennent aussi les devants à l'association. Carol Couture, adjoint à l'Archiviste au Service des archives de 1972 à 1976 et directeur de 1976 à 1988, est membre de l'association depuis les années 70. II est d'abord impliqué à la Section de gestion des documents de Montréal (1974), puis au Comité des structures professionnelles (1975). Il joint la revue Archives en 1976 et la retrouve à la fin des années 90 comme directeur (1999-2000, 2000-2001). II siège également au conseil d'administration comme président à trois reprises (1979-1980, 1988-1989, 2012-2013). Au cours de sa présidence, I'AAQ revoit sa structure avec l'engagement d'un directeur général (1988), met en place de procédures pour la gestion courante et insiste sur la rigueur dans la gestion des réunions. Pour toute son implication, qui continue à ce jour, Carol Couture reçoit le titre de membre émérite en 1991.

Jacques Ducharme, chef de la division des archives historiques de 1973 jusqu'en 1981, offre son expertise à l'AAQ depuis les années 70 sur divers comités et projets. Au conseil d'administration, il est conseiller (1972-73, 1979-1980) et trésorier (1973-1974). En son rôle de trésorier, il s'assure que l'AAQ engage un vérificateur externe et met en place des méthodes comptables efficaces. II est également secrétaire-trésorier pour la Section des archives historiques de la région de Montréal (1974) et collaborateur à la revue Archives de 1976 à 1981, notamment sous le rôle de rédacteur en chef. En 1979, il prend en charge la direction de la Section des archives historiques. II est donc en poste lorsque les archivistes religieux, un peu désillusionnés de leur place à I'association, décident de créer le Regroupement des archivistes religieux (RAR). II signe avec le nouveau regroupement une entente permettant aux archivistes religieux de demeurer au sein de l'AAQ. Cette division au sein de l'association amène un nouveau questionnement sur sa structure. C'est d'ailleurs Jacques Ducharme qui préside le comité d'orientation mis en place pour redéfinir le rôle et la mission de l'AAQ (Baillargeon et Lévesque, 2007-2008, p.2). 
La transition entre la décennie 80 et la suivante s'oriente dans la résolution de problèmes d'organisation. Un secrétariat permanent est mis en place pour permettre une gestion plus efficace. Les questionnements sur le nom de l'association et la désignation des membres comme archiviste continuent (Association des archivistes du Québec, 1980). La majorité des membres sentent une difficulté à créer un sentiment d'appartenance commune en lien avec des valeurs fondamentales entre chacun des secteurs représentés par l'association. Le travail du comité d'orientation mené par Jacques Ducharme amène I'AAQ à changer ses sections basées sur le milieu auquel l'archiviste appartient vers une appartenance régionale (1988). Cette dissolution des sections pour la constitution de cinq regroupements régionaux (1990) ouvre les années 90 pour l'AAQ (Association des archivistes du Québec, 2016a).

Un moment important pour la communauté archivistique dans les années 90 est la venue à Montréal, en 1992, de la Conférence internationale des archives. Claude Minotto prend la direction du comité organisateur de cet événement majeur; comité créé dès 1987 puisque ce congrès qui a lieu aux quatre ans n'est pas une mince affaire. Pour aider le comité dans sa charge, une Commission nationale canadienne du Congrès est mise sur pied. Cet organisme consultatif réunit des représentants du Conseil canadien des archives, de l'Association canadienne des archivistes, de l'Association des archivistes du Québec, des Archives provinciales du Canada, de la Ville de Montréal, et quelques grandes entreprises privées (Minotto, 1995). Le Canada accueille pour la première fois ce congrès et avec 2700 participants venus de tous les continents, cet événement est « une vitrine exceptionnelle pour l'archivistique québécoise » (Bibliothèque et Archives nationales du Québec, s. d.).

\section{LE PRÉSENT DE L'AAQ}

La fin des années 90 et le début des années 2000 voient une progression stable de l'association, qui reste tout de même à l'affût des besoins d'amélioration de sa structure. En 2004, la région Centre est abolie; I'AAQ se divise maintenant en quatre sections régionales (Association des archivistes du Québec, 2016a).

Un projet qui rassemble la communauté durant cette période est la Déclaration québécoise sur les archives. Ce projet mené par Denys 
Chouinard, chef de la Division des archives historiques de 1981 à 1990, fait son chemin avec le lancement de la Déclaration en 2006, puis la déclaration québécoise devient la Déclaration universelle sur les archives (septembre 2010) endossée par I'UNESCO à Paris en novembre 2011.

À cette aventure participe une autre employée de la DGDA. Diane Baillargeon, directrice du Service depuis 2009, travaille sur les comités de I'AAQ en lien avec la Déclaration soit le groupe de travail de rédaction et le comité de promotion de la Déclaration québécoise et le groupe de travail sur la rédaction d'une Déclaration universelle.

Diane Baillargeon est une bénévole impliquée pleinement à l'association depuis 1989. Membre du comité des affaires professionnelles, membre des comités organisateurs de quelques congrès, elle est également présidente à la fin des années 90 (1997-1998). Pour souligner sa contribution, elle reçoit le prix de la présidence en 2003 et le titre de membre émérite en 2012 (Association des archivistes du Québec, 2016b).

Le quotidien de l'AAQ reste ancré dans ses comités permanents. On y retrouve toujours plusieurs bénévoles également employés de la DGDA. Michel Champagne, archiviste, est impliqué dans la revue Archives depuis 1991 et directeur de celle-ci depuis 2006. Pour son rôle dans l'évolution de la revue Archives, il reçoit le prix du président en 2012 et le titre de membre émérite en 2014. Nathalie Denis, archiviste également, est partie prenante du comité de la région de Montréal de 2001 à 2012 en tant qu'administratrice, trésorière et directrice. Elle participe aussi au comité du site Web de 2001 à 2006 comme membre puis responsable du comité (2003-2006). Sous sa gouverne, le comité voit à la création de l'interface Web de la revue Archives (version 2003) et à la refonte du site Web (version 2005). Ce site Web lancé dans sa première version au milieu des années 90 est un projet dirigé par Yves Lapointe, alors contractuel à la DGDA, qui se voit remettre le prix du président en 1997. Nathalie Denis reçoit elle aussi le prix du président en 2004 et est vice-présidente en 2007-2008. Taïk Bourhis, responsable des systèmes de gestion à la DGDA, a été membre du sous-comité de la bibliographie de la revue Archives et membre du comité du site Web. Elle a également reçu le prix du meilleur article de la revue Archives pour le texte Le projet Hippocampe: mise en place d'un système de gestion électronique de documents à l'Université de Montréal en 2011. La nouvelle génération n'est pas en reste avec les techniciennes Julie Contant et Noura Elmobayed-Langevin, toutes deux impliquées dans le comité de direction de la région Montréal. 
En ce début de XXle siècle, le désir de renouveau et d'innovation reste au cœur de l'association. En 2012, le conseil d'administration approuve la création du rôle de représentant des membres étudiants pour s'assurer de conserver un dynamisme du côté de la relève. Les partenariats sont toujours une partie importante de la force de l'AAQ, démontrée par la signature d'une entente avec le Réseau des services d'archives du Québec en 2014 (Association des archivistes du Québec, 2015) et avec I'Association des archivistes canadiens en 2015 (Association des archivistes du Québec, 2014).

\section{CONCLUSION}

La Division de la gestion de documents et des archives de I'Université de Montréal a toujours été étroitement liée à l'Association des archivistes du Québec par les personnes qui les ont toutes deux servies. Ce qui montre la place importante, voire centrale, de la DGDA en particulier, mais des services d'archives universitaires en général pour l'archivistique québécoise.

Tenter de délimiter en trois grandes périodes les liens entre la DGDA et l'AAQ est une chose difficile, presque impossible, ce qui démontre bien la loyauté de ces bénévoles et leur passion pour leur association. Une autre tâche difficile est de tenter de synthétiser en quelques mots l'accomplissement complet de chacun de ceux-ci dans le milieu archivistique, vous comprendrez donc que plusieurs autres personnalités, faits et détails ont été écartés.

Pour terminer, une question: Pourquoi s'impliquer? Dans les mots de Louise Gagnon-Arguin "parce qu'on croit à l'importance d'une association dans la promotion et la reconnaissance de la profession» (Gagnon-Arguin, 2015). Cet exercice a démontré qu'en fait, il n'y a jamais eu de moment stable ou de certitude paisible pour l'association et, conséquemment, pour les archivistes eux-mêmes. Ce qui supporte encore plus l'idée de la nécessité de rester alerte; la nécessité d'avoir une force d'impact, même si le contexte est différent des débuts de l'AAQ. Une force d'action est toujours nécessaire; I'implication et le support de tous sont donc d'autant plus vitaux. II ne nous reste plus qu'à nous inspirer des bénévoles présentés ici pour assurer un autre demi-siècle aussi dynamique au milieu de l'archivistique québécoise. 


\section{BIBLIOGRAPHIE}

AAQ membre émérite. (1981). Fonds François Beaudin (P674, 1999-05003/21). BAnQ Vieux-Montréal, Montréal, Québec.

ASSOCIATION DES ARCHIVISTES DU QUÉBEC. (1975). Actes du

$3^{e}$ congrès annuel tenu à l'Université de Montréal et à l'auberge Le Vieux St-Gabriel les 2 et 3 mai 1974. Fonds François Beaudin (P674, 1999-05-003/21). Montréal. BAnQ Vieux-Montréal, Montréal, Québec.

ASSOCIATION DES ARCHIVISTES DU QUÉBEC. (1980). Procès-verbal de I'Assemblée générale annuelle. 8 mai 1980.

ASSOCIATION DES ARCHIVISTES DU QUÉBEC. (2014). Nouvelle entente AAQ et ACA - AAQ - Association des archivistes du Québec. Repéré à http://www.archivistes.qc.ca/nouvelles/nouvelles-aaq/975-nouvelleentente-aaq-aca

ASSOCIATION DES ARCHIVISTES DU QUÉBEC. (2015). Entente entre I'AAQ et le RAQ - AAQ - Association des archivistes du Québec.

ASSOCIATION DES ARCHIVISTES DU QUÉBEC. (2016a). Historique - AAQ - Association des archivistes du Québec.

ASSOCIATION DES ARCHIVISTES DU QUÉBEC. (2016b). Prix annuels - AAQ - Association des archivistes du Québec.

BAILLARGEON, D. et LÉVESQUE M. (2007-2008). L'Association des archivistes du Québec, une association impliquée, des archivistes engagés. Archives 39(1), 43-92.

BEAUDIN, F. (1976). Rapport du président pour l'année 1975-1976. Archives 8(2), 19-23.

BEAUDIN, F. (1982). Née à Québec... Mais conçue à Santa Fe, New Mexico (USA)... et à Québec! Décembre 1982. La Chronique XII(4).

BIBLIOTHÈQUE ET ARCHIVES NATIONALES DU QUÉBEC. (sans date). Historique | BAnQ. Repéré à http://www.banq.qc.ca/a_propos_banq/ historique 
BIRON, L.-A. (1972). Pourquoi je suis archiviste. Archives 4(2), 16-24.

CHOQUET, L. (1982). Hommage aux fondateurs de l'Association des archivistes du Québec 1967-1982. La Chronique XII(4), 2-4.

COMITÉ DE FORMATION DE L'ASSOCIATION DES ARCHIVISTES DU QUÉBEC. (15 mai 1971). Rapport sur la formation des archivistes. Fonds François Beaudin (P674, 1999-05-003/36) BAnQ Vieux-Montréal, Montréal, Québec.

CONSEIL DE L'ASSOCIATION DES ARCHIVISTES DU QUÉBEC. (1974). Réunion du 25 octobre 1974. Fonds François Beaudin (P674, 199905-003/20). BAnQ Vieux-Montréal, Montréal, Québec.

COUTURE, C. (1992). La normalisation en archivistique: un pas de plus dans l'évolution d'une discipline: mélanges Jacques-Ducharme. Québec: Documentor.

GAGNON-ARGUIN, L. (1992a). L'archivistique. Son histoire, ses acteurs depuis 1960. Sainte-Foy, Québec: Presses de I'Université du Québec.

GAGNON-ARGUIN, L. (1992b). L'association des archivistes du Québec, Vingt-cing ans d'histoire. Archives 24(1-2), 9-24.

GAGNON-ARGUIN, L. (2015). Carol Couture et la vie associative. Dans L. GAGNON-ARGUIN, et M. LAJEUNESSE, (dir.), Panorama de I'archivistique contemporaine: évolution de la discipline et de la profession; Mélanges offerts à Carol Couture (p. 37-42). Québec: Presses de I'Université du Québec

GARON, R. (1974). L'association des archivistes du Québec. Archives 6 (2), 5-10.

LESSARD, C. (1982). L'AAQ après quinze ans, quelques propos d'un ancien président. La Chronique XII(4) Décembre 1982, 6.

MINOTTO, C. (1978). Rapport de Claude Minotto, secrétaire. Archives $10(2), 24-25$.

MINOTTO, C. (1995). De Montréal à Beijing. Impacts du Congrès international des archives. Archives 26(4), 9-27. 
Partage des tâches entre le conseil d'administration et le conseil exécutif. (31 mai 1974). Fonds François Beaudin (P674, 1999-05-003/20, chemise: AAQ Conseil 1974/1975). BAnQ Vieux-Montréal, Montréal, Québec.

SOCIETY OF AMERICAN ARCHIVISTS. (2016). Annual Meeting Locations and Attendance | Society of American Archivists. Repéré à http:// www2.archivists.org/conference/annual-meeting-locations-andattendance\#60s

UNIVERSITÉ DE MONTRÉAL. DIVISION DE LA GESTION DE DOCUMENTS ET DES ARCHIVES. (2010). Répertoire des cadres de I'Université de Montréal 1877-2012. Repéré à http://www.archiv.umontreal.ca/ publication/index.html 